\title{
Modeling and Optimization of Collaborative Passenger Control in Urban Rail Stations under Mass Passenger Flow
}

\author{
Lili Wang, Xuedong Yan, and Yun Wang \\ State Key Laboratory of Rail Traffic Control and Safety, Beijing Jiaotong University, Beijing 100044, China \\ Correspondence should be addressed to Xuedong Yan; xdyan@bjtu.edu.cn
}

Received 29 July 2014; Revised 30 October 2014; Accepted 10 November 2014

Academic Editor: Hong Chen

Copyright ( 2015 Lili Wang et al. This is an open access article distributed under the Creative Commons Attribution License, which permits unrestricted use, distribution, and reproduction in any medium, provided the original work is properly cited.

\begin{abstract}
With the rapid development of urban rail transit, the phenomenon of outburst passenger flows flocking to stations is occurring much more frequently. Passenger flow control is one of the main methods used to ensure passengers' safety. While most previous studies have only focused on control measures inside the target station, ignoring the collaboration between stops, this paper puts emphasis on joint passenger control methods during the occurrence of large passenger flows. To provide a theoretic description for the problem under consideration, an integer programming model is built, based on the analysis of passenger delay and the processes by which passengers alight and board. Taking average passenger delay as the objective, the proposed model aims to disperse the pressure of oversaturated stations into others, achieving the optimal state for the entire line. The model is verified using a case study and the results show that restricted access measures taken collaboratively by stations produce less delay and faster evacuation. Finally, a sensitivity analysis is conducted, from which we find that the departure interval and maximum conveying capacity of the train affect passenger delay markedly in the process of passenger control and infer that control measures should be taken at stations near to the one experiencing an emergency.
\end{abstract}

\section{Introduction}

Congestion problems in China's large cities have created an urgent need for the construction and expansion of the metro system. However, in some exceptional circumstances (such as in peak hours or with a large increase, or outburst, of passenger flow), passenger pressure exceeds the range that the facilities can bear. Mass passenger flow brings about an increase in safety risks, a reduction in passenger comfort, and the extension of travel times. As a result, many researchers have focused on the study of large passenger flows.

Studies of pedestrian characteristics in crowded situations are important for research on passenger evacuation. In fact, such research has always paid attention to the simulation of pedestrians' microscopic behavior. Cheung and Lam researched pedestrian flow characteristics [1], route choice [2], and simulation models in MTR (mass transit railway) stations in Hong Kong [3]. Harris improved the microtrips model to simulate passenger behavior in urban rail stations in London [4], and then created the SCM (station congestion model) to simulate the effects of passenger delays on a variety of station facilities. Daamen and Hoogendoorn constructed the software NOMAD for microscopic model simulation capable of predicting the impact of facility properties on passenger volumes, travel times, and waiting times at stations [5]. Mahudin et al. described the development of an instrument that could capture the dimensionality of rail passenger crowding and its relationship to the experience of stress and feelings of exhaustion [6]. There have also been some other studies of pedestrians' microscopic behavior $[7,8]$.

When considering the impact of a large passenger flow on an urban rail station, the alighting and boarding processes of passengers should not be ignored. Many metro stations have been suffering from high levels of pedestrian density of late [9]. Several statistical analyses of accident data, performed in China, Europe, and the United States [10-13], show that many injuries occur in the boarding and alighting processes. Exiting studies about these processes in urban rail stations have focused on observation and modeling. Heinz measured 


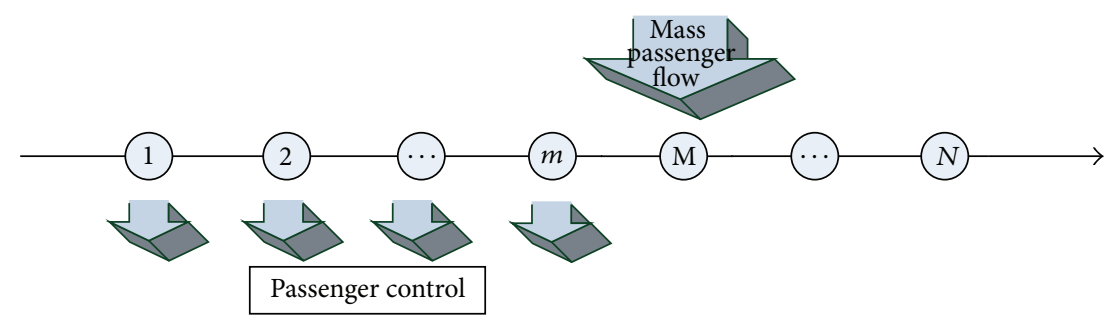

FIGURE 1: Schematic view of collaborative passenger control.

the alighting and boarding times for different trains [14]. $\mathrm{Xu}$ and $\mathrm{Wu}$ developed velocity-density mathematical models for Shanghai metro stations [15]. Furthermore, a three-layer architecture-adaptive agent model was proposed by Xu Qi to simulate passengers' microscopic behaviors in complex passenger flow on an urban rail transit platform [16].

All these studies are theoretically enlightening for the solving of problems relating to mass passenger flow. In practice, to prevent the occurrence of safety accidents, many measures have been taken, such as the adjustment of the stop-schedule plan, changes in the train routing scheme, and the control of passenger flow [17]. Among these measures, passenger flow control serves as the main method, one which sacrifices the station service level to ensure security. Thus, flow-limiting measures are temporarily taken in these oversaturated rail transit stations to avoid the excessive pressure caused by large passenger flows. Most studies have focused on control measures only for oversaturated stations. For example, Bauer et al. conducted a simulation of macroscopic pedestrian flow for the design of crowd control measures for public transport after the occurrence of special events [18]. However, in practice, passenger control measures are, more extensively, divided into three levels, namely, the station level, the line level, and the regional level [19]. At the station level, only oversaturated stations have these kinds of measures applied, such as passenger control on the platform, stairs, or at the entrance to the station, which is helpful for limiting the number of passengers on the platform but is not effective for achieving rapid evacuation. At the line and regional level, control measures are also applied to other stations where appropriate, to relieve the traffic pressure on a particular station in an emergency situation. Unfortunately, detailed strategies for this method have not been proposed yet. For example, there are no further researches concerning which stations should be controlled and how many people are allowed in. This will be addressed in the present study.

To disperse traffic pressure on an oversaturated station and allocate the transport capacity rationally, this paper proposes a programming model to determine the control parameters in specific stations and to show the effect of collaborative control measures. In the modeling process, the following issues are addressed: (1) analytical formulations for computing passenger delay over the entire line; (2) analysis of passengers' alighting and boarding procedure; and (3) optimal solutions considering the delays to all passengers.

The remainder of this paper is organized as follows. The overall problem statement and assumptions are presented in
Section 2. In Section 3 we construct a programming model for passenger flow optimization. The numerical analysis of the model for verifying the rationality of this model is presented in Section 4, where the sensitivity analysis is conducted to illustrate how the key factors influence passengers' delay in the related stations. In the last section, we draw conclusions and discuss some potential research topics for the future.

\section{Problem Statement}

This study deals with an urban rail line on which station $\mathrm{M}$ suffers the occurrence of mass passenger flow. Passengers gather with a common purpose, which makes the quantity and whereabouts of people entering station $\mathrm{M}$ predictable. In this paper, we consider the mass passenger flow to occur in one direction, with passenger volume $Z$. As is shown in Figure 1 , there are $m$ stops before station $M$. Besides the passenger flow control in station $\mathrm{M}$, we take control measures on the preceding $m$ stops as the mass passenger flow enters station $\mathrm{M}$, which will reduce the number of people who can get on the train until all passengers in station $M$ have been evacuated.

In the actual control process, what station managers want to know is the proportion of passengers moving onto the platform, as well as the duration for which traffic is limited, so that they can take measures including restricted access at the entrance and on the passages, escalators, and so forth. Therefore, the decision variables in the model are the number of passengers moving onto the platform in a particular period during the time for which traffic is limited, which is set to $y_{j}$, and the number of time periods $n$.

Several points should be noted. (1) This paper considers mass passenger flow in a certain direction of a rail line, while it may occur in both directions or even across several lines in reality. However, passenger flow in each direction and for every line can be calculated, so the model is applicable to such realities. (2) The passenger control measure is also taken in station $\mathrm{M}$, but is not considered in this model, because it is only helpful for limiting the number of passengers moving onto the platform and not for evacuating rapidly and decreasing the delay. However, once the number of people boarding at station $\mathrm{M}$ has been obtained using this model, it will help to determine the entry rate to the oversaturated station. (3) Obviously, the earlier the measures are taken, the more rapidly the emergency will be processed. Therefore, 
the program will be started as soon as the mass passenger flow begins. (4) As the passenger flow in a metro station changes periodically in coordination with the train departure intervals, the entire evacuation time is divided into periods that begin with the departure of the previous train and end with the departure of the next.

In order to facilitate the model formulation, other assumptions used throughout the paper are as follows: (1) for each rail transit (except at station $M$ ), the number of passengers moving onto the platform in unit time throughout the control process is certain, including the number of people arriving at or transferring at the station; (2) during the time for which traffic is limited, the train departure interval is held constant; (3) the total number of passengers in the unusually large flow (termed here the outburst flow) in a certain direction is predictable.

\section{Modeling}

3.1. The Basic Idea. In period $i$, when a train is running on the line, the maximum conveying capacity, that is, the maximum number of people who can be transported per unit of time, is constant. Considering the general case, let the stops ahead be equivalent to one, and when the train arrives at station $\mathrm{M}$ and passengers get off, let the number of people on the train be $A_{i}$, the number getting onto the train be $B_{i}$, and the maximum conveying capacity of the train be $E$. The following equation is clearly established:

$$
A_{i}+B_{i} \leq E .
$$

In the control case, when the train arrives at station $M$ and passengers alight, let the number of people on the train be $C_{i}$ and the number boarding the train $D_{i}$. Therefore, similarly,

$$
C_{i}+D_{i} \leq E \text {. }
$$

Whether we limit the passenger flow or not, the maximum conveying capacity of a train is constant, but the key is the adjustment to the values of $C_{i}$ and $D_{i}$. Thus, the state of the whole line under the impact of an outburst in traffic will be used to achieve the overall optimum.

As for the specific control scheme, the main idea is shown in Figure 2, and the model will be expanded accordingly.

As is shown in Figure 2, there are mass passenger flows occurring in the target station $M$ which result in the passenger delay of the whole line. We divided stations in the line into three groups, namely, stations before $M$, station $M$, and stations after $\mathrm{M}$. To minimize the passenger delay of the whole line, collaborative passenger control measures are conducted in stations before $M$ and station $M$ during the evacuation process, in which we pay attention to passengers stranded, alighting, and boarding. In the course of passenger control, there are some constraints including conveying capacity of train, the number of delivered of passengers, controlled passengers, and the length of passenger control time. In addition, the capacity of the target station is considered as a restrictive condition. However, it is a parameter for calculating the passenger entry rate in the target station according to the number of boarding passengers.

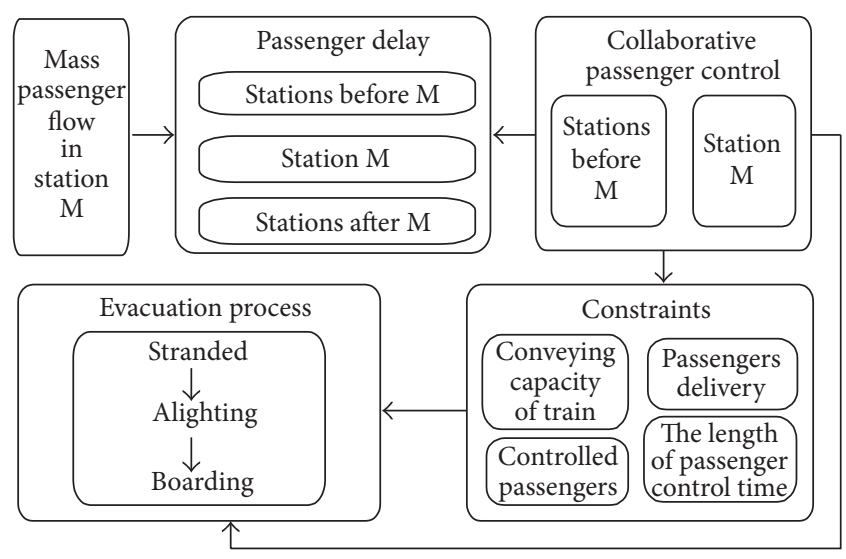

Figure 2: Main idea of the passenger control model.

3.2. Analysis of Passenger Delay. The most direct consequence of a large passenger flow is the passenger delay, which is what this model aims to minimize. To analyze the impact on passengers, the entire line is seen as a system. Under passenger control of the preceding $m$ stops, passenger delay $T$ caused by the outburst in passenger flow is generated at all stops along the line. Meanwhile, without passenger control, passenger delay $T^{\prime}$ is generated at stops from $\mathrm{M}$ to the terminal. If $T^{\prime}>T$, it will make sense to control the passenger flow of the preceding $m$ stops. Therefore, the model aims to minimize the passenger delay of the whole system.

In the situation where the traffic must be limited, the passenger delay $T$ is divided into three parts, which are labeled $T_{1}, T_{2}$, and $T_{3}$ respectively. In this study, $T_{1}$ is defined as the delay time for the passengers in the stations before the target station, which will be expected to increase during control; $T_{2}$ is defined as the delay time for the passengers in the target station, which will be expected to decrease during control; $T_{3}$ is defined as the delay time for all of the passengers in the stations after the target station, which is influenced by the large passenger flow and passenger control measures.

For the preceding $m$ stops, $a_{i j}$ and $a_{i j}^{\prime}$ are defined as the number of passengers boarding the train at station $j$ in period $i$ under normal and control circumstances, respectively, $t$ as the train departure interval, and $n$ as the number of periods over which the passenger control occurs. Then, the passenger delay at the preceding $m$ stops can be expressed as in the following equation:

$$
T_{1}=\sum_{i=1}^{n} \sum_{j=1}^{m}\left(a_{i j}-a_{i j}^{\prime}\right) *(n+1-i) * t .
$$

For station $\mathrm{M}$, let $d_{i}^{\prime}$ be the number of passengers boarding the train in period $i$ under control circumstances. Then, the passengers' delay at station $M$ can be expressed as in the following equation:

$$
T_{2}=\sum_{i=1}^{n}\left(d_{i}^{\prime} *(i-1) * t\right) .
$$

For the stations after $\mathrm{M}$, of which there are $l$, let $c_{i k}$ and $c_{i k}^{\prime}$ be the number of passengers boarding the train at station $k$ 
in period $i$ under normal and control circumstances respectively. Then, the passengers' delay at the stations after M can be expressed as in the following equation:

$$
T_{3}=\sum_{k=1}^{l} \sum_{i=1}^{n}(n+1-i) *\left(c_{i k}-c_{i k}^{\prime}\right) * t
$$

3.3. Analysis of Passengers' Alighting and Boarding. During the evacuation process, the state of a station changes as passengers alight and board. Take the preceding $m$ stops during normal circumstances as an example. The number of passengers boarding equals the minimum of the number of passengers on the platform and the surplus capacity of the train. In the early stages of a given period, the number of passengers stranded equals the number of people remaining when the train left in the previous period, and the number of people on the train depends on the number of people already aboard at the previous station, and the numbers alighting and boarding. The above procedure can be expressed as in the following equation:

$$
\begin{gathered}
a_{i j}=\min \left(x_{j}+p_{i j}, E-\left(1-h_{j}\right) * u_{i j}\right), \\
p_{i j}=x_{j}+p_{i-1, j}-a_{i-1, j}, \\
u_{i j}=\left(1-h_{j}\right) * u_{i, j-1}+a_{i, j-1},
\end{gathered}
$$

where, $a_{i j}$ denotes the number of passengers boarding the train at station $j$ in period $i ; p_{i j}$ denotes the number of passengers stranded at station $j$ in period $i$; $u_{i j}$ denotes the number of people on the train at station $j$ in period $i ; x_{j}$ denotes the number of people moving onto the platform in a given period at station $j ; E$ denotes the maximum conveying capacity of the train; $h_{j}$ denotes the off-rate of station $j$, which means the rate at which passengers alight from the train.

The calculation can be conducted in a similar way when it comes to the process during the control situation and for the stations from $M$ to the terminal. However, because the functions contain a recursive process, it is necessary to set initial values. Obviously, the number of people on the train at the origin stop is zero, that is, $u_{i 1}=0$. In addition, the numbers of passengers stranded on the platform in all of the stations are also needed.

\subsection{Constraints and Objective Function}

3.4.1. Conveying Capacity Constraints. As expressed in (7), the number of people boarding at station $\mathrm{M}$ can be no more than the surplus capacity of the train:

$$
\left(\left(1-h_{j}\right) * u_{i m}^{\prime}+a_{i m}^{\prime}\right) *(1-r)+d_{i}^{\prime} \leq E
$$

where $u_{i m}^{\prime}$ and $a_{i m}^{\prime}$ denote the number of passengers on and boarding the train at station $m$ in period $i$ under control circumstances, respectively, and $r$ denotes the off-rate in station M.
3.4.2. Passenger Delivery Constraints. As (8) shows, the outburst of passengers needs to be conveyed within the $n$ periods:

$$
\sum_{i=1}^{n} d_{i}^{\prime} \geq\left(Z+n * s+p_{\mathrm{M}}\right)
$$

where $p_{M}$ denotes the number of passengers stranded at station $\mathrm{M}$ in the first period, and $s$ denotes the number of people moving onto the platform in a given period in station $\mathrm{M}$, besides the outburst of passengers.

3.4.3. Constraints to Passengers Moving onto the Platform. Based on the effects of this model, the number of passengers moving onto the platform at the preceding $m$ stops under control circumstances should not exceed that in normal conditions, as (9) shows:

$$
y_{j}<x_{j}
$$

3.4.4. Constraints on the Length of Time for Which Passenger Controls Are Imposed. Two extreme cases are envisaged. Firstly, all passengers at the preceding $m$ stops are prohibited from moving onto the platform. In this case, the outburst of passenger flow can be conveyed as quickly as possible. Let the number of periods be $n_{\min }$. Secondly, the preceding $m$ stops proceed without control measures. In this case, the outburst of passenger flow will take the longest amount of time to be conveyed. Let the number of periods be $n_{\max }$. The corresponding function is shown in the following equation:

$$
n_{\min } \leq n \leq n_{\max }
$$

3.4.5. Objective Function. The goal of the model is to minimize the average passenger delay across the whole line, which is represented by $T_{a}$ in the following equation:

$$
\begin{aligned}
\min T_{a}= & \left(T_{1}+T_{2}+T_{3}\right) \\
& \times\left(\sum_{j=1}^{m}\left(x_{j} * n+p_{1 j}\right)+\sum_{k=1}^{l}\left(f_{k} * n+q_{1 k}\right)\right. \\
& \left.+g_{1}+Z+n * s\right)^{-1} .
\end{aligned}
$$

The latter part of the formula denotes the number of passengers affected across all the stations on the line throughout the process. Respectively, $x_{j}$ and $p_{1 j}$ are the number of people moving onto the platform and stranded at stations before $M$, while $f_{k}$ and $q_{1 k}$ have similar meanings for the stations after M. Meanwhile, $g_{1}$ represents the number of people stranded on platform at station $\mathrm{M}$.

\section{Numerical Analysis}

4.1. A Simple Case. We present a simple case with an urban rail line on which a station named $\mathrm{M}$ suffers from an outburst of passenger flows in a certain direction, numbering 8000 
TABLE 1: Input data for stations before $\mathrm{M}$.

\begin{tabular}{cccc}
\hline Station $j$ & $\begin{array}{c}\text { The number of people } \\
\text { entering the platform in } \\
\text { one period, } x_{j}\end{array}$ & Off-rate $h_{j}$ & $\begin{array}{c}\text { The number of } \\
\text { people stranded } \\
\text { initially, } p_{1, j}\end{array}$ \\
\hline 1 & 200 & 0.2 & 50 \\
2 & 400 & 0.3 & 50 \\
3 & 300 & 0.5 & 50 \\
\hline
\end{tabular}

TABLE 2: Input data for stations after M.

\begin{tabular}{lccc}
\hline $\begin{array}{l}\text { Station } \\
k\end{array}$ & $\begin{array}{c}\text { The number of people } \\
\text { moving onto the platform } \\
\text { in one period, } f_{k}\end{array}$ & Off-rate $w_{k} \begin{array}{r}\text { The number of } \\
\text { people stranded } \\
\text { initially, } q_{1, k}\end{array}$ \\
\hline 1 & 300 & 0.45 & 50 \\
2 & 400 & 0.2 & 50 \\
3 & 200 & 0.3 & 50 \\
4 & 400 & 0.5 & 50 \\
\hline
\end{tabular}

TABLE 3: Optimal solution.

\begin{tabular}{lccc}
\hline & Under control & Without control & Saving \\
\hline Average delay (min) & 9.18 & 10.01 & 0.83 \\
Number of periods & 8 & 10 & 2 \\
Evacuation time $(\mathrm{min})$ & 40 & 50 & 10 \\
\hline
\end{tabular}

people. Control measures at the preceding three stops are taken to provide greater conveying capacity for station $\mathrm{M}$.

In this case, the train departure interval $t$ is set to 5 minutes using data from the thesis [20] which researched on passenger flow forecasting of urban rail transit, and the maximum conveying capacity of train $\mathrm{E}$ is 1500 according to the thesis [21] which concerned about the issue of optimal deployment of passenger capacity. At station $\mathrm{M}$, the number of people moving onto the platform in one period during normal circumstances, $s=300$, the off-rate $r=0.3$, and the number of passengers stranded on the platform is 50 . Other input data are given in Table 1 . The remaining data are assumed basing on the passenger flow data in the research [16] which simulated passenger flows on urban rail transit platform.

For the stations after $M$, the input data are given in Table 2. The resulting passenger control parameters are listed in Table 3, as obtained using LINGO, which shows that taking passenger control measures reduces the delay and the evacuation time and demonstrates the feasibility of this model. Table 4 shows the change of the three kinds of delays under control and without control. Compared to the situation without control, Delay of passengers under control in stations before $\mathrm{M}$ increases $6.8 \mathrm{~min}$ averagely, while it decreases $8.2 \mathrm{~min}$ and $1.3 \mathrm{~min}$ averagely in station $\mathrm{M}$ and stations after $\mathrm{M}$, respectively. Delay of passengers in station $\mathrm{M}$ decreases significantly under control. For the preceding three stops, the number of people moving onto the platform in one period under control compared with normal circumstances is shown in Table 5. In this case, stations 1 and 2 are proceeding without passenger control measures, while station 3 allows

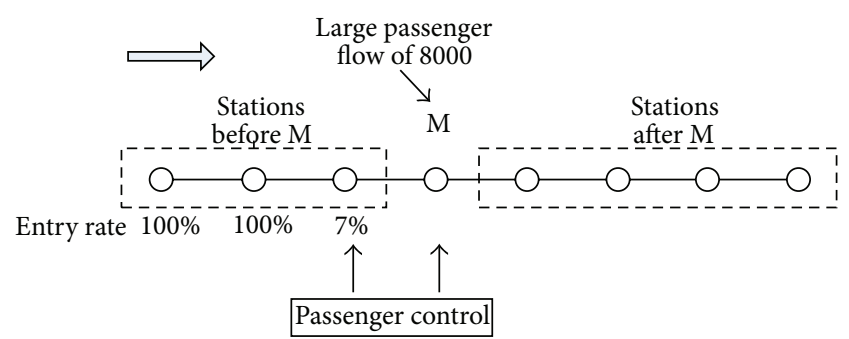

FIGURE 3: Schematic of case study.

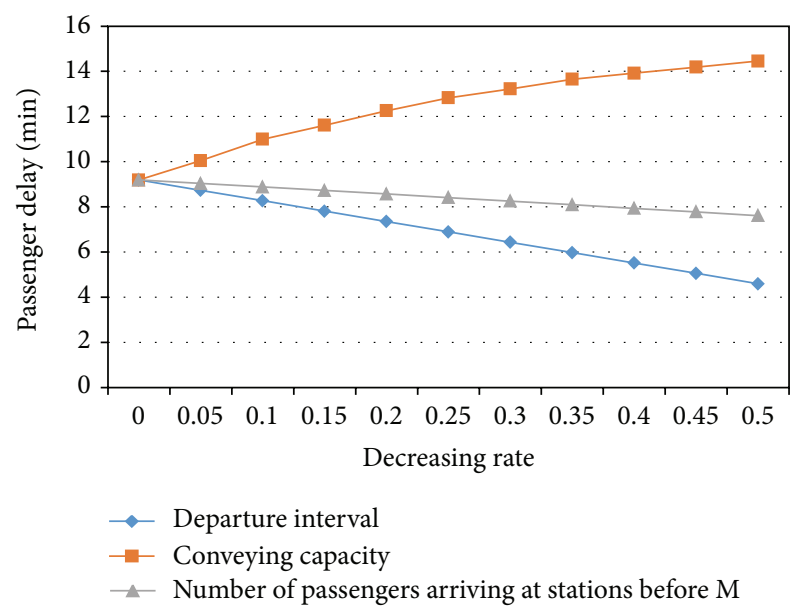

FIGURE 4: Sensitivity analysis of average passenger delay.

$7 \%$ of passengers to move onto the platform. The station managers are assumed to take appropriate measures aimed at minimizing the passenger delay over the whole line. The description and main results are shown in Figure 3.

\subsection{Sensitivity Analysis}

4.2.1. Sensitivity Analysis of Key Factors. In this part, sensitivity analysis will be conducted on the model to identify the factors that have the greatest impact on the line in terms of passenger delay. The model shows that the main factors affecting the delay are the departure interval and the maximum capacity of the train, and the number of passengers arriving at the stations before M. Sensitivity analysis of these three factors is carried out to enable optimizing strategies to be proposed.

We observe the changing trend of average passenger delay first of all. As shown in Figure 4, factors including departure intervals of the train and number of passengers arriving at the stations before $M$ decrease with a rate of $\Delta p=5 \%$, which makes the passengers' average delay, represented by the value of the ordinate, decrease gradually. However, as the decrease of maximum conveying capacity of train, delay increases. The whole figure shows the greater impacts of the departure interval and maximum conveying capacity of the train. Therefore, to further optimize the average passenger delay, the following strategies are expected to be effective. 
TABLE 4: Comparison of passenger delays.

\begin{tabular}{|c|c|c|c|c|c|c|c|c|}
\hline \multirow[b]{2}{*}{ Station } & \multicolumn{3}{|c|}{ Under control } & \multicolumn{3}{|c|}{ Without control } & \multirow[b]{2}{*}{$\begin{array}{l}\text { Change of } \\
\text { average delay } \\
\quad(\mathrm{min})\end{array}$} & \multirow[b]{2}{*}{$\begin{array}{c}\text { Change of } \\
\text { total delay } \\
\text { (min) }\end{array}$} \\
\hline & $\begin{array}{c}\text { The number of } \\
\text { influenced } \\
\text { passengers }\end{array}$ & $\begin{array}{l}\text { Total delay } \\
\quad(\min )\end{array}$ & $\begin{array}{l}\text { Average delay } \\
\text { (min) }\end{array}$ & $\begin{array}{c}\text { The number of } \\
\text { influenced } \\
\text { passengers }\end{array}$ & $\begin{array}{l}\text { Total delay } \\
\quad(\mathrm{min})\end{array}$ & $\begin{array}{l}\text { Average delay } \\
\text { (min) }\end{array}$ & & \\
\hline Stations before $\mathrm{M}$ & 7350 & 50220 & 6.8 & 9150 & 0 & 0 & 6.8 & 50220 \\
\hline Station M & 10450 & 183402 & 17.6 & 11000 & 283348 & 25.8 & -8.2 & -99946 \\
\hline Stations after M & 10600 & 27084 & 2.6 & 13200 & 50600 & 3.8 & -1.3 & -23516 \\
\hline All the stations & 28400 & 260706 & 9.2 & 33350 & 333948 & 10 & -0.8 & -73242 \\
\hline
\end{tabular}

TABLE 5: Control parameters in the optimal situation.

\begin{tabular}{lccc}
\hline Station $j$ & $x_{j}$ & $y_{j}$ & Entry rate \\
\hline 1 & 200 & 200 & $100 \%$ \\
2 & 400 & 400 & $100 \%$ \\
3 & 300 & 21 & $7 \%$ \\
\hline
\end{tabular}

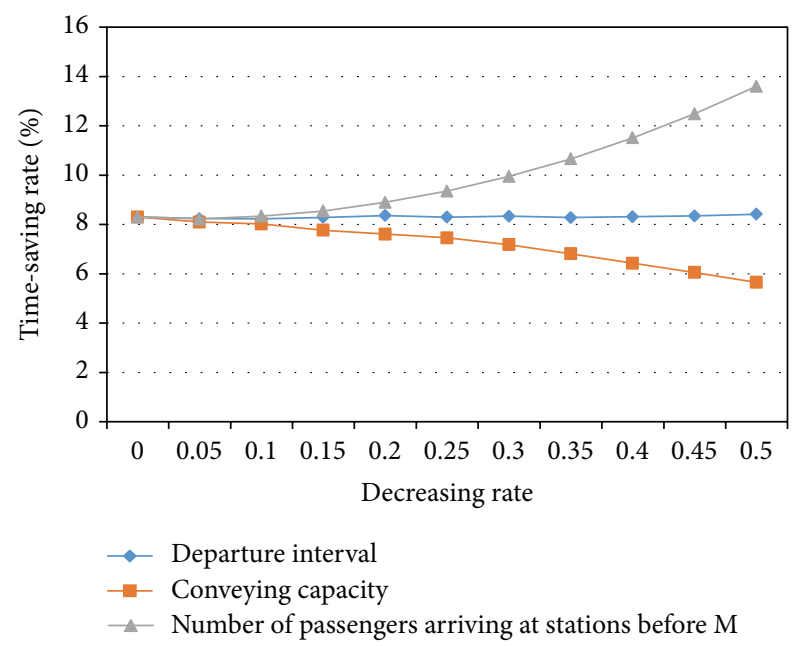

FIGURE 5: Sensitivity analysis of time-saving rate.

(1) On the basis of normal station operations, the departure interval of trains should be shortened moderately.

(2) Larger trains should be used to increase the carrying capacity.

Time-saving rate represents the ratio of average saving time of passengers on the line with control measure to the delay time without control. Figure 5 shows that time-saving rate is essentially unchanged with the decrease of departure interval while it decreases as conveying capacity of train decreases and increases as passenger flow decreases. In the analysis, the maximum saving time could be up to $2.52 \mathrm{~min}$ under the condition that departure interval is $5 \mathrm{~min}$, conveying capacity of train is 1500 and the number of passengers arriving at stations before $\mathrm{M}$ is 100,200 , and 150 , respectively.

4.2.2. Sensitivity Analysis of Controlled Stations. To probe the characteristics of controlled stations, sensitivity analysis is

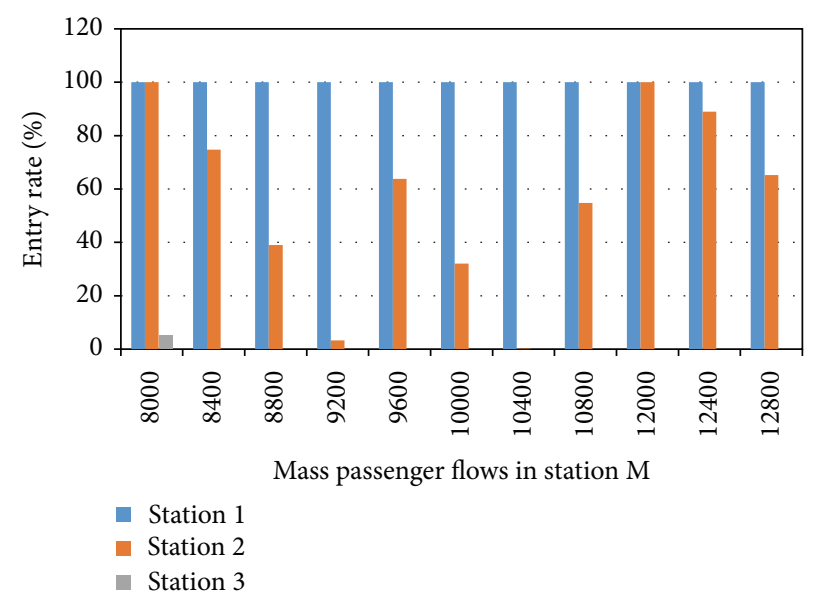

FIgURE 6: Sensitivity analysis of controlled stations with increasing passenger flows in station $\mathrm{M}$.

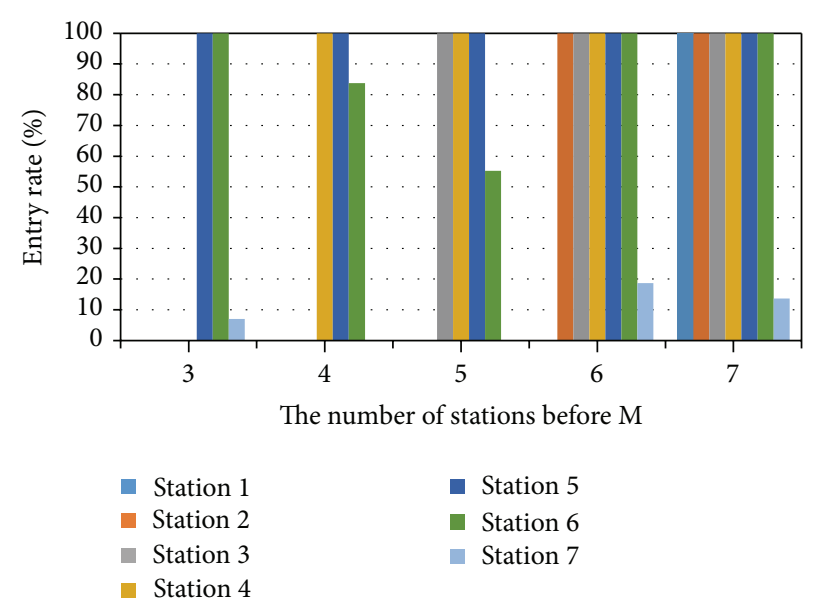

FIGURE 7: Sensitivity analysis of controlled stations conducted by adding stations before $\mathrm{M}$.

conducted to observe changes in the entry rates, the rate at which they are allowed onto the platform, at the three stations before $\mathrm{M}$ during optimal situations, by increasing the mass passenger flow at station $\mathrm{M}$ and adding stations before $\mathrm{M}$. The results are shown in Figures 6 and 7.

From Figure 6, we find that the closer a station is to $\mathrm{M}$, the lower will be the arrival rate, which means that 
stations near to $\mathrm{M}$ should take more control measures. More specifically, the stations further away from $M$ are controlled only if passengers are forbidden to enter the nearer stations. This conclusion is more obvious in Figure 7 when the number of stations before $\mathrm{M}$ is increased gradually. When the collaborative passenger control program is conducted to relieve the pressure of the outburst of passenger flow, closer stations should take action first, which will help to reduce the delay.

\section{Conclusions}

Focusing on a station with an outburst of passenger flow, this paper presents an integer programming model that aims to minimize the passenger delay integrally and evacuate passengers as soon as possible by taking control measures at other appropriate stops. Based on the analysis of the passengers' delay across all stations and the process by which passengers alight and board trains, the relationship between stations as well as periods is clarified. Taking average passenger delay as the objective, the proposed model disperses the pressure on an oversaturated station among other stations, achieving the optimal state of the entire system.

This model has been successfully tested through a case study, and the results show that the proposed method can effectively solve the passenger control problem of an urban rail line with an outburst of passengers. Furthermore, sensitivity analysis of the model has been conducted. Firstly, we identified the factors having the greatest impact on the line in terms of passenger delay, under passenger control conditions, the results of which showed that the departure interval and maximum conveying capacity of the train were the most influential. Secondly, we probed the characteristics of the controlled stations, showing that stations near to the one experiencing the outburst should take the lead in implementing control measures. This is of practical relevance for station managers. A future study will consider passenger control methods in the case of more than one oversaturated station.

The method can be put into effect in practice. Specifically, when a station suffers large passenger flow in the case of an emergency, passenger control measures are taken in the upstream adjacent stations. This model can be applied to identify the number of passengers being allowed to enter the platforms and the entry rates of the controlled stations. The passenger control in a station can be realized through placing barriers in the station channels, reducing the number of ticket-checking gates or even temporarily closing the station. These measures are supposed to be taken coherently according to the number of controlled passengers.

Additionally, there are also other approaches in practice to manage the traffic in urban rail transportation, such as price control. Compared to passenger control measure, price control is inclined to affect travel demand of passengers for whole network, which is more appropriate for long-term effect of balance traffic distributions between rail lines or between rail networks and road networks. The two methods can be combined for both dynamic management and demand adjustment of passenger flows in the urban rail networks.

It should be noted that since the numbers of passengers in the controlled stations are restricted, the passengers' delays would increase to a certain degree, which may lower the levels of service for the controlled stations. However, through adjustment of passenger traffic distribution in stations and rail network, the passenger control countermeasure can relieve the imbalance of passenger flows. From the perspective of safety, the passengers in the target station that suffers large passenger flow can be evacuated more rapidly, which reduces the possibility of accidents caused by overload of stations' facilities. From the perspective of system optimization, the average delay for all passengers in the rail network will be significantly reduced.

\section{Conflict of Interests}

The authors declare that there is no conflict of interests regarding the publication of this paper.

\section{Acknowledgments}

This work is financially supported by the Chinese National 973 Project (2012CB725403), Chinese National Natural Science Foundation (71210001), and the State Key Laboratory of Rail Traffic Control and Safety (RCS2012ZT009). The study is also supported by the Center of Cooperative Innovation for Beijing Metropolitan Transportation.

\section{References}

[1] C. Y. Cheung and W. H. K. Lam, "A study of the bi-directional pedestrian flow characteristics in the Hong Kong mass transit railway stations," Journal of Transportation Engineering, vol. 2, no. 5, pp. 1607-1619, 1997.

[2] C. Y. Cheung and W. H. K. Lam, "Pedestrian route choices between escalator and stairway in MTR stations," Journal of Transportation Engineering, vol. 124, no. 3, pp. 277-285, 1998.

[3] J. Y. S. Lee, W. H. K. Lam, and S. C. Wong, "Pedestrian simulation model for Hong Kong underground stations," in Proceedings of the IEEE Intelligent Transportation Systems, pp. 554-558, IEEE, Oakland, Calif, USA, August 2001.

[4] N. G. Harris and R. J. Anderson, "An international comparison of urban rail boarding and alighting rates," Proceedings of the Institution of Mechanical Engineers F: Journal of Rail and Rapid Transit, vol. 221, no. 4, pp. 521-526, 2007.

[5] W. Daamen and S. P. Hoogendoorn, "Experimental research of pedestrian walking behavior," Transportation Research Record, no. 1828, pp. 20-30, 2003.

[6] N. D. M. Mahudin, T. Cox, and A. Griffiths, "Measuring rail passenger crowding: scale development and psychometric properties," Transportation Research Part F: Traffic Psychology and Behaviour, vol. 15, no. 1, pp. 38-51, 2012.

[7] S. P. Hoogendoorn and W. Daamen, "Design assessment of Lisbon transfer stations using microscopic pedestrian simulation," in Computers in Railways IX (Congress Proceedings of CompRail 2004), Dresden, Germany, pp. 135-147, WIT Press, Southampton, UK, 2004. 
[8] S. P. Hoogendoorn and W. Daamen, "Pedestrian behavior at bottlenecks," Transportation Science, vol. 39, no. 2, pp. 147-159, 2005.

[9] X. Y. Xu, J. Liu, H. Y. Li, and J. Q. Hu, "Analysis of subway station capacity with the use of queueing theory," Transportation Research Part C. Emerging Technologies, vol. 38, pp. 28-43, 2014.

[10] M. Abril, F. Barber, L. Ingolotti, M. A. Salido, P. Tormos, and A. Lova, "An assessment of railway capacity" Transportation Research Part E: Logistics and Transportation Review, vol. 44, no. 5, pp. 774-806, 2008.

[11] M. T. Baysari, A. S. McIntosh, and J. R. Wilson, "Understanding the human factors contribution to railway accidents and incidents in Australia," Accident Analysis \& Prevention, vol. 40, no. 5, pp. 1750-1757, 2008.

[12] E. Ben-Elia and D. Ettema, "Rewarding rush-hour avoidance: a study of commuters' travel behavior," Transportation Research Part A. Policy and Practice, vol. 45, no. 7, pp. 567-582, 2011.

[13] M. Kyriakidis, R. Hirsch, and A. Majumdar, "Metro railway safety: an analysis of accident precursors," Safety Science, vol. 50, no. 7, pp. 1535-1548, 2012.

[14] W. Heinz, Passenger service times on trains-theory, measurements and models [Ph.D. thesis], Royal Institute of Technology, Stockholm, Sweden, 2003.

[15] W. Xu and Z. Wu, "Mathematical model for passenger flow in subway station waiting rooms," Journal of Railway Science and Engineering, pp. 70-75, 2005.

[16] Q. Xu, B. Mao, M. Li, and X. Feng, "Simulation of passenger flows on urban rail transit platform based on adaptive agents," Journal of Transportation Systems Engineering and Information Technology, vol. 14, no. 1, pp. 28-33, 2014.

[17] W. O. Assis and B. E. A. Milani, "Generation of optimal schedules for metro lines using model predictive control," Automatica, vol. 40, no. 8, pp. 1397-1404, 2004.

[18] D. Bauer, S. Seer, and N. Brändle, "Macroscopic pedestrian flow simulation for designing crowd control measures in public transport after special events," in Proceedings of the Smmer Computer Simulation Conference, pp. 1035-1042, Society for Computer Simulation International, 2007.

[19] L. Qiao, Transport organization of the outburst large passenger flow in urban rail transit [M.S. thesis], Beijing Jiaotong University, Beijing, China, 2013.

[20] W. Lina, Passenger flow forecasting and scheduling research of urban rail transit based on the historical data [Master thesis], Beijing Jiaotong University, Beijing, China, 2011.

[21] S. Wenwen, Study on short-time passenger flow forecast and the optimal capacity allocation problem in urban mass transit [Master thesis], Beijing Jiaotong University, Beijing, China, 2011. 


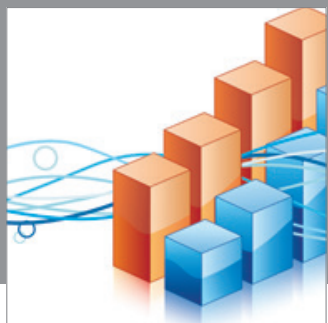

Advances in

Operations Research

mansans

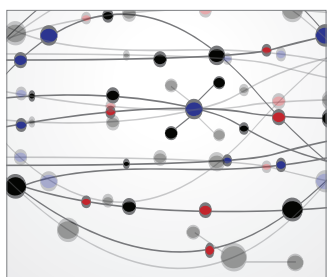

The Scientific World Journal
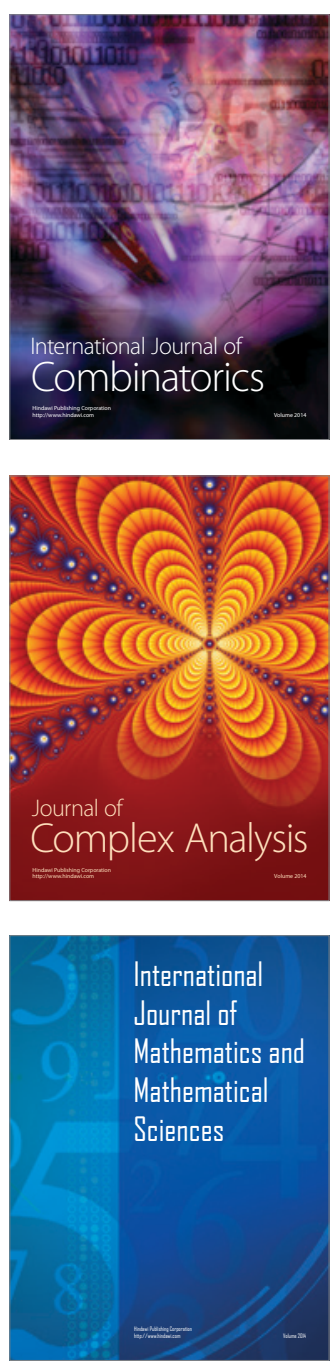
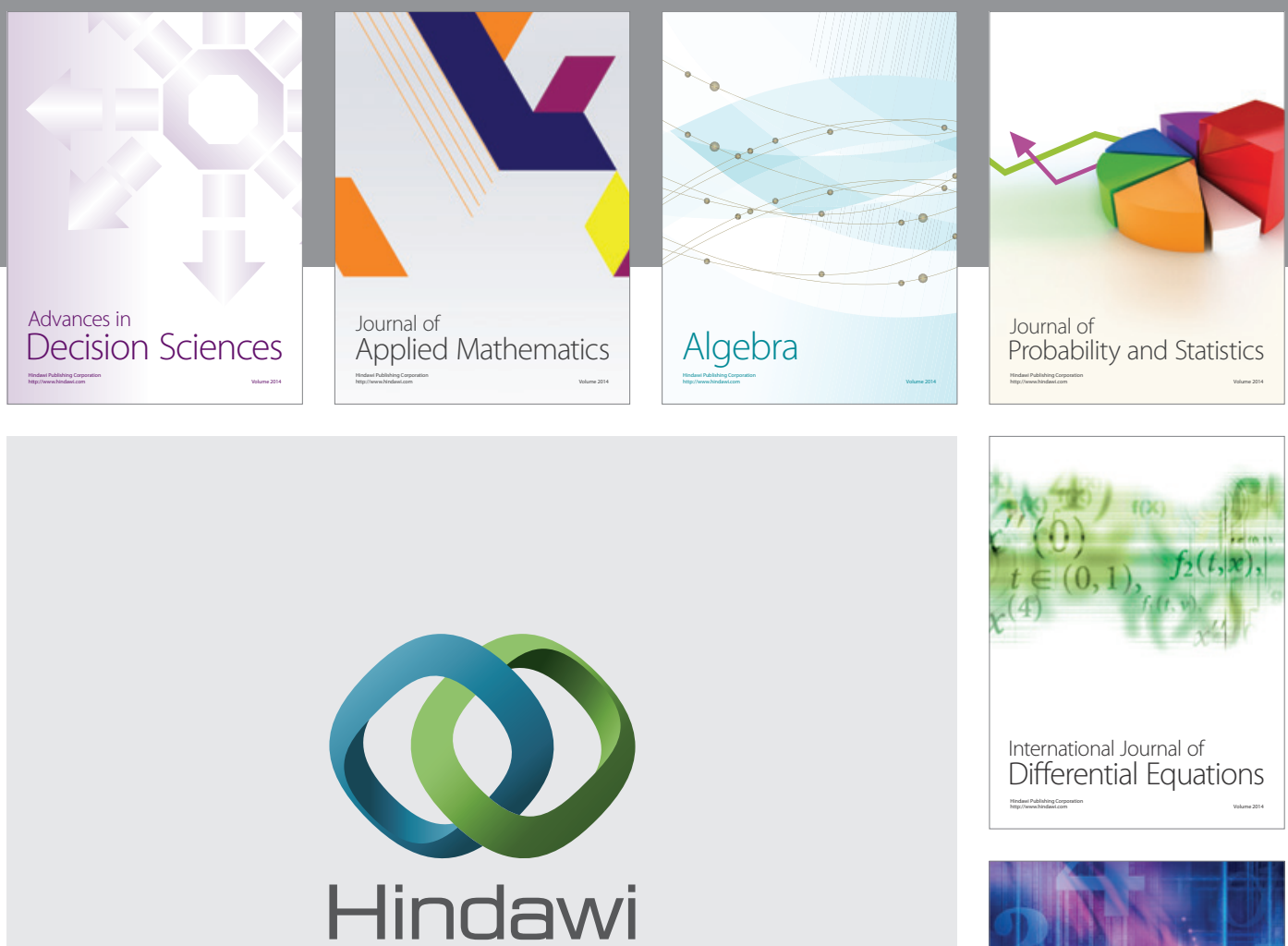

Submit your manuscripts at http://www.hindawi.com
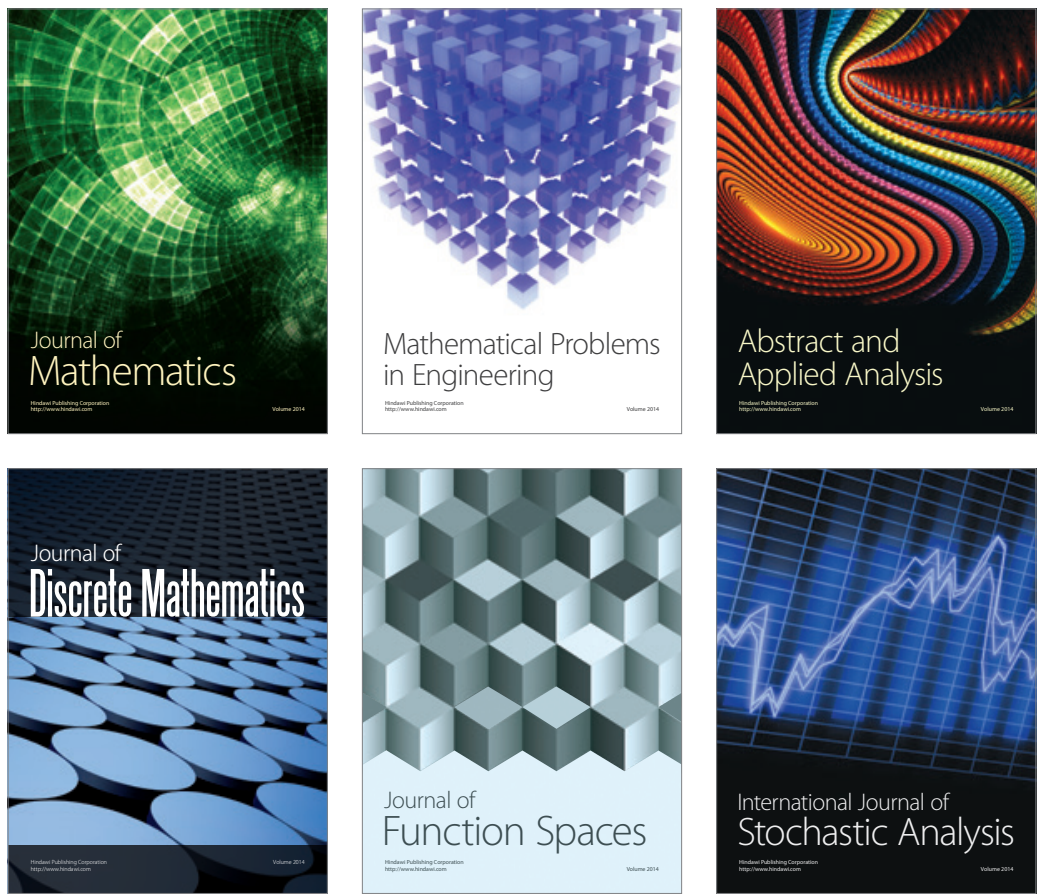

Journal of

Function Spaces

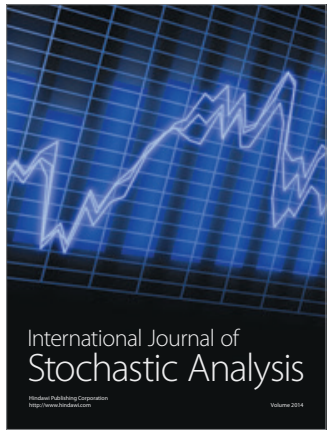

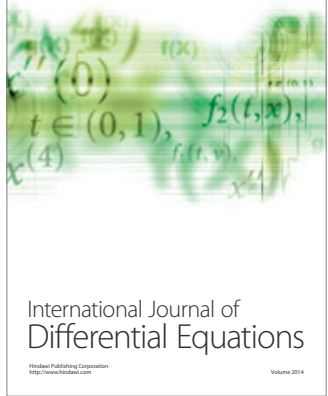
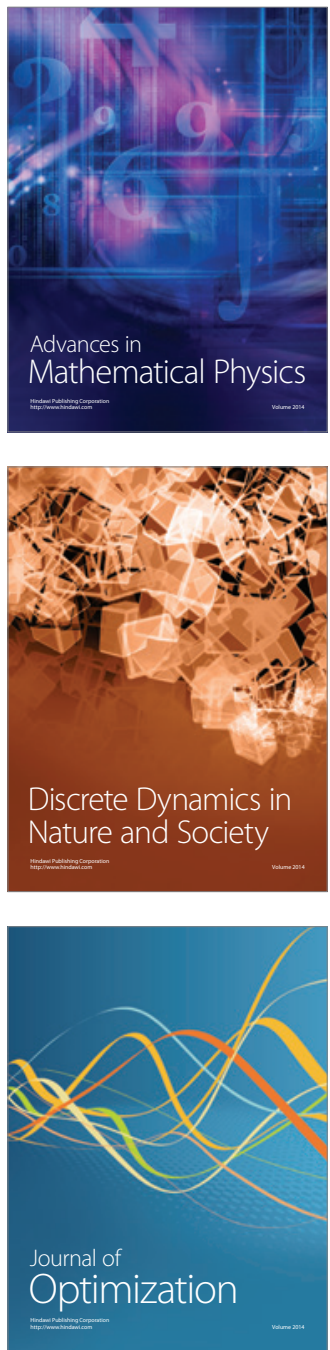